\title{
AN EXTREMAL PROBLEM FOR QUASICONFORMAL MAPPINGS IN AN ANNULUS ${ }^{1}$
}

\author{
ALVIN M. WHITE
}

\begin{abstract}
The following extremal problem is solved. We consider a family of continuously differentiable univalent quasiconformal mappings $w=f(z)$ of the annulus $r<|z|<1$ onto the unit disk minus some continuum containing the origin. For a point $b$ on a fixed circle, maximize $|f(b)|$ within the family.

The problem is solved by using a variational method due to Schiffer. The extremal function and the maximum are found in terms of the Weierstrass $\wp$-function and the elliptic modular function.
\end{abstract}

In his investigation of numerical construction of conformal mappings, D. Gaier [3] considered the family $F$ of functions $f(z)$ regular, analytic and univalent in the annulus $R: r<|z|<1$ and satisfying the following three conditions:

$$
|f(z)|<1 \quad \text { in } R \text { while }|f(z)|=1 \text { on }|z|=1 ;
$$

that is, $f(z)$ maps $R$ onto the unit disk minus some continuum $\Gamma$;

$$
\begin{gathered}
f(z) \neq 0 \text { in } R,(\Gamma \text { contains the origin }) ; \\
f(1)=1,(\text { a normalization }) .
\end{gathered}
$$

Gaier raised the question of finding the maximum of $|f(z)-z|$ for all $f \in F$ and $z \in R$. Duren and Schiffer [2] solve this problem by a method of variation within the family $F$. (See also Gehring and Hällström [5] and Gaier and Huckemann [4].)

In order to answer the question raised by Gaier, Duren and Schiffer first solve the extremal problem:

$$
\max |f(b)|, \quad f \in F, \quad \text { where }|b|=r_{1}, \quad r<r_{1}<1 .
$$

This problem is of independent interest. Grötzsch [6] previously solved it by the method of extremal length.

In the present paper the requirement of analyticity is weakened and problem (*) is solved within a family $F^{\prime}$ of quasiconformal mappings by a variational method.

The author would like to thank Professor Schiffer for suggesting the topic

Presented to the Society, January 17,1972 ; received by the editors July 27,1972 and, in revised form, December 6, 1977.

AMS (MOS) subject classifications (1970). Primary 30A38; Secondary 30A60.

${ }^{1}$ This research is partially supported by NSF. The author thanks the referee for helpful suggestions.

○ American Mathematical Society 1978 
and for his encouragement and help during the preparation of this paper.

1. Let $w=f(z)=u(x, y)+i v(x, y)$ be a family of continuously differentiable univalent mappings of a domain $D_{z}$ in the $z$ plane onto a domain $D_{w}$ in the $w$ plane.

We call the mapping $K$-quasiconformal in $D_{z}$ if it satisfies the inequality ${ }^{2}$

$$
\begin{gathered}
\left|f_{z}\right|^{2}+\left|f_{\bar{z}}\right|^{2} \leqslant K\left(\left|f_{z}\right|^{2}-\left|f_{\bar{z}}\right|^{2}\right) ; \quad K \geqslant 1, \\
\left|\frac{f_{\bar{z}}}{f_{z}}\right|^{2} \leqslant \frac{K-1}{K+1}
\end{gathered}
$$

for all points of $D_{z}$. Note that

$$
\left|f_{z}\right|^{2}+\left|f_{\bar{z}}\right|^{2}=\frac{1}{2}\left(u_{x}^{2}+u_{y}^{2}+v_{x}^{2}+v_{y}^{2}\right)
$$

and

$$
\left|f_{z}\right|^{2}-\left|f_{\bar{z}}\right|^{2}=u_{x} v_{y}-u_{y} v_{x}=\partial(u, v) / \partial(x, y)
$$

is the Jacobian of the mapping [1].

Denote the family $f(z)$ of univalent, class $C^{1}$ functions satisfying (1), (2), (3), (4) as $F^{\prime}$. Consider the extremal problem:

$$
\max |f(b)|, \quad f \in F^{\prime} \quad \text { where }|b|=r_{1}, \quad r<r_{1}<1 .
$$

Problem (7) is solved by a variational method. In the variational method we seek that function (if it exists) in the family for which the functional $\chi[f]=|f(b)|$ attains its maximum value. The value of the functional is compared for functions of the family which are near each other in a certain sense. A comparison function within the family $F^{\prime}$ is constructed by a method of Schiffer [12], [2]. The existence of the extremal function can be shown as in [12], [10].

Let $w=f(z)$ be a given function in $F^{\prime}$ and let $\Gamma$ denote the complement within the open disk of the range $\Delta(f)$ of $f$. Let $w_{0} \neq 0$ be a fixed point on the continuum $\Gamma$. Corresponding to each point $w_{0}$ it is known [2] that there exist variation functions $V_{\rho}(w)$ analytic and univalent in the range of $f$ having the following properties:

(i) $\lim _{\rho \rightarrow 0} V_{\rho}(w)=w$;

(ii) $\left|V_{\rho}(w)\right|=1$ for $|w|=1$;

(iii) $V_{\rho}(w) \neq 0$ for $w$ in $\Delta(f)$;

(iv) $V_{\rho}(1)=1$.

Such a function is

$V(w)=w\left[1+\frac{a \rho^{2}(1-w)}{\left(w-w_{0}\right)\left(1-w_{0}\right) w_{0}}+\frac{\bar{a} \rho^{2}(1-w)}{\left(1-\bar{w}_{0} w\right)\left(1-\bar{w}_{0}\right) \bar{w}_{0}}+0\left(\rho^{3}\right)\right]$

for proper choice of the coefficient $a$. A comparison function in the class $F^{\prime}$ is

$$
f^{*}(z)=V_{\rho}[f(z)]
$$

\footnotetext{
${ }^{2}$ This is not the currently customary definition.
} 
Suppose $f(z)$ is the extremal function, and denote $f(b)=B$. We have $\left|V_{\rho}[f(b)]\right| \leqslant|f(b)|$. Introducing (8) this yields

$$
\left|1+\frac{a \rho^{2}(1-B)}{\left(B-w_{0}\right)\left(1-w_{0}\right) w_{0}}+\frac{\bar{a} \rho^{2}(1-B)}{\left(1-\bar{w}_{0} B\right)\left(1-\bar{w}_{0}\right) \bar{w}_{0}}+0\left(\rho^{3}\right)\right| \leqslant 1 .
$$

Noting that $\operatorname{Re}\{\alpha\}=\operatorname{Re}\{\bar{\alpha}\}$, and $|B|<1$, a calculation yields

$$
\operatorname{Re}\left\{\frac{a \rho^{2}}{w_{0}\left(B-w_{0}\right)\left(1-w_{0} \bar{B}\right)}+0\left(\rho^{3}\right)\right\} \leqslant 0 .
$$

2. From the fundamental lemma of boundary variation [11] in the theory of conformal mapping, we can conclude from inequality (11), which is valid for all admissible variations, that $\Gamma$ is an analytic arc $w=w(t)$ satisfying the differential equation

$$
\frac{\left(w^{\prime}\right)^{2}}{w(B-w)(1-w \bar{B})}>0
$$

The analytic arc $\Gamma$ must pass through the origin since $f(z) \neq 0$ in $R$.

Obviously the ray $w=B t, 0 \leqslant t \leqslant 1$, satisfies (12) and contains the origin. Because of the uniqueness theorem for the differential equation [2], this segment is the only solution containing the origin and hence $\Gamma$ must be a part of it.

Thus we prove that the extremal function $f$ maps the annulus $R$ onto the unit disk slit along a segment starting at the origin in the direction of $B$.

3. Without loss of generality we can assume that $B>0$. Indeed, let $B=|B| e^{i \beta}$ and let $\alpha$ be defined by $f\left(e^{i \alpha}\right)=e^{i \beta}$. Then the function $\varphi(z)=$ $e^{-i \beta} f\left(e^{i \alpha} z\right)$ also belongs to $F^{\prime}$ and maps $R$ onto the disk slit along the real axis and takes the value $|B|$ on the circle $|z|=|b|$.

4. In order to characterize the extremal function $f(z)$ another variation will be introduced. We choose a point $w_{0}$ in the range of $f$ and define a variation $\tilde{V}_{\rho}(w)$ which has the form (8) for $\left|w-w_{0}\right| \geqslant \rho$, but must be modified inside the disk to remain of class $C^{1}$.

$$
\begin{array}{r}
\tilde{V}_{\rho}(w)=w\left[1+\frac{a(1-w)}{w_{0}\left(1-w_{0}\right)} \cdot\left(\overline{w-w_{0}}\right)\left(2-\frac{\left|w-w_{0}\right|^{2}}{\rho^{2}}\right)\right. \\
\left.+\frac{\bar{a} \rho^{2}(1-w)}{\left(1-\bar{w}_{0} w\right)\left(1-\bar{w}_{0}\right) \bar{w}_{0}}+0\left(\rho^{3}\right)\right]
\end{array}
$$

satisfies the requirement. An easy calculation shows that our functional $\chi[f]=|f(b)|$ satisfies the asymptotic equation 


$$
\chi\left[f^{*}\right]=\chi[f]\left[1+\operatorname{Re} \frac{a \rho^{2}\left(1-B^{2}\right)}{w_{0}\left(B-w_{0}\right)\left(1-B w_{0}\right)}+0\left(\rho^{3}\right)\right]
$$

since we may assume that for sufficiently small $\rho, B$ is outside the disk $\left|w-w_{0}\right| \leqslant \rho$.

The assumption that $f(z)$ is the extremal function for our problem implies that $\chi\left[f^{*}\right] \leqslant \chi[f]$ for all variations $\tilde{V}_{\rho}[w]$ which preserve the $K$-quasiconformality of the varied function. This condition allows the application of [12]. It is seen that the extremal function $w=f(z)$ satisfies the relation

$$
J(w)-\sqrt{K-1 / K+1} \overline{J(w)}=k(z)
$$

where

$$
J(w)=\int_{1}^{w} \frac{d w}{\sqrt{w(B-w)(1-w B)}}, \quad w=f(z)
$$

and $k(z)$ is analytic. Note that $k(z)$ is multivalued and has a singularity at $b$.

The extension (13) of (8) into $\left|w-w_{0}\right|<\rho$ may lack a uniformity property that is needed in [12] as was pointed out by Renelt [10]. This is remedied by using the solution of a Beltrami equation as shown in [14]. The rest of the procedure remains unchanged.

We can transform (15) so as to express $J(w)$ in terms of $k(z)$ and $\overline{k(z)}$.

$$
J(w)=\frac{K+1}{2} k(z)+\frac{1}{2} \sqrt{K^{2}-1} \overline{k(z)} .
$$

Differentiating $\left(15^{\prime}\right)$ with respect to $x$ yields

$$
\frac{1}{\sqrt{w(B-w)(1-B w)}} f_{x}=\frac{K+1}{2} k^{\prime}(z)+\frac{1}{2} \sqrt{K^{2}-1} \overline{k^{\prime}(z)} \text {. }
$$

Let us consider that point on $|z|=r$ which corresponds to the right endpoint of the slit $\Gamma$. Obviously, at that point the Jacobian of the mapping vanishes and hence $f_{z}=0$. This means that $k^{\prime}(z)$ has a zero on the circle $|z|=r$.

5. In order to study the boundary behavior of the analytic function $k(z)$, we observe that the circumference $|z|=1$ is mapped by $f(z)$ onto $|w|=1$. On $|w|=1$ we can write

$$
d J(w)=\frac{d w}{\sqrt{w(B-w)(1-B w)}}=\frac{i e^{i \alpha} d \alpha}{\sqrt{e^{i \alpha}\left(B-e^{i \alpha}\right)\left(1-B e^{i \alpha}\right)}}
$$

where $w=e^{i \alpha}$. Hence

$$
d J(w)=\frac{i d \alpha}{\sqrt{\left(B-e^{i \alpha}\right)\left(e^{-i \alpha}-B\right)}}=\frac{d \alpha}{\left|B-e^{i \alpha}\right|}=\text { real. }
$$

In view of (15) we can assert that $d k(z)$ is real for $|z|=1$.

Next consider the image of $|z|=r$ which we have shown to be a segment of the positive real axis. Since this segment does not reach the point $B$ which 
corresponds to $b(r<|b|<1), J(w)$ is real on that segment and so is $k(z)$ in view of (15).

Since we can parametrize $z=e^{i \alpha}$ and $z=r e^{i \alpha}$ on the two circumferences, we have in both cases $d z=i z d \alpha$ and from the observation that $k^{\prime}(z) d z=$ real in each case, we infer that on each circumference

$$
l(z)=z^{2} k^{\prime}(z)^{2} \leqslant 0 .
$$

From the definition of $k(z)$ it is easily seen that $k^{\prime}(z)$ is unbounded in the neighborhood of $z=b$, while $\sqrt{z-b} k^{\prime}(z)$ is regular.

Since $l(z)$ is real for $|z|=r$ and $|z|=1$, by the Schwarz reflection principle, we have

$$
l(1 / \bar{z})=\overline{l(z)}=l\left(r^{2} / \bar{z}\right)
$$

Hence $l\left(r^{2} z\right)=l(z)$. Now, let $t=\log z, L(t)=l(z)$. Then, in the rectangle

$$
\log r \leqslant \operatorname{Re}\{t\}<\log 1 / r, \quad 0 \leqslant \operatorname{Im}\{t\}<2 \pi
$$

the function $L(t)$ is regular analytic except for two simple poles at $t_{1}=\log b$ and $t_{2}=\log 1 / \bar{b}$. It is doubly periodic

$$
L(t+2 \log r)=L(t), \quad L(t+2 \pi i)=L(t) .
$$

In view of (17), $L(t)$ is negative for $\operatorname{Re}\{t\}=0$ and $\operatorname{Re} t=\log r$. Consider the half period parallelogram

$$
\log r<\operatorname{Re}\{t\} \leqslant 0, \quad \gamma \leqslant \operatorname{Im} t \leqslant \gamma+\pi
$$

where $b=|b| e^{i \gamma}$. We can map this parallelogram onto the upper half-plane such that the point $\log b$ on its boundary goes into infinity. The mapping function $\psi(t)$ is easily seen to be doubly periodic with the same periods as $L(t)$ and with the same poles. Hence, by the well-known uniqueness theorem for elliptic functions

$$
L(t)=A \psi(t)+C .
$$

Since $\psi(t)$ and $L(t)$ are both real for $\operatorname{Re}\{t\}=0$ and $\operatorname{Re}\{t\}=\log r$, clearly $A$ and $C$ are real constants. We also see that $L(t)$ gives a one-to-one map of the boundary of the half period parallelogram onto the real axis. Hence, it has precisely one simple zero which corresponds to the zero of $k^{\prime}(z)$ on the circle $|z|=r$. We know by (17) that $l(z)$ is nonpositive for those $z$ corresponding to the vertical lines of the parallelogram. Hence, its simple zero must be a corner point: either $[\log r+i \gamma]$ or $[\log r+i(\gamma+\pi)]$. Observe that the only other point on the boundary where $L(t)$ can change its sign is the point $\log b$ where it has a simple pole. Thus we infer that on the boundary of the half period parallelogram $L(t)$ is positive on the segment $\langle\log r+i \gamma, \log |b|+i \gamma\rangle$ and is negative on the rest.

Returning to the $z$-plane, we find that $z^{2} k^{\prime}(z)^{2}$ is negative for $z=s e^{i \gamma}$ with $|b|<s<1$. Now,

$$
\frac{d}{d s} k\left(e^{i \gamma} s\right)=e^{i \gamma} k^{\prime}\left(e^{i \gamma} s\right)
$$

and hence we can conclude that $d / d s k\left(e^{i \gamma} s\right)$ is imaginary for this interval. 
Thus $k(b)-k\left(e^{i \gamma}\right)=$ imaginary. On the other hand, observe that $J(B)$ is pure imaginary and so is, by $(15), k(b)$. Hence, we conclude $k\left(e^{i \gamma}\right)=$ imaginary. Finally, $J(w)$ is real for $|w|=1$ by $(16)$, whence $k\left(e^{i \gamma}\right)=0$. By $\left(15^{\prime}\right)$ the image $w$ of $e^{i \gamma}$ satisfies $J(w)=0$. By $(16), J(1)=0$. By the normalization, the point 1 corresponds to 1 . Hence, $e^{i \gamma}=1$, and we infer $b>0$.

It has been shown that $L(t)$ vanishes at $\log r+i \gamma$ and that $\gamma=0$. This implies that $k^{\prime}(r)=0$.

6. Now we complete the argument as follows: $k(z)$ is pure imaginary on the real axis between $b$ and 1 . It follows from (15') and (16) that $w=f(z)$ is real on that segment. On the segment between $r$ and $b$ we see that $l(z)>0$, which implies $\left(k^{\prime}(z) z\right)^{2}>0$. Hence $k^{\prime}(z) z$ and $k^{\prime}(z)$ are real on that segment. Thus $\left(15^{\prime \prime}\right)$ leads to the differential equation

$$
\frac{d w}{\sqrt{w(B-w)(1-w B)}}=\text { real. }
$$

This implies that $w=f(z)$ is real between $r$ and $b$.

We conclude that $f(z)$ maps the inner circumference $|z|=r$ onto the continuum $\Gamma$ which is a segment of the positive axis. The point $z=-r$ is mapped into $w=0$ and $z=r$ into the right end of the slit.

Thus the single valued analytic function $\left[z k^{\prime}(z)\right]^{2}$ is negative on the circumferences $|z|=1$ and $|z|=r$, has a simple pole at $z=b$, and vanishes at $z=r$.

By Rouche's theorem it is easy to see that this function takes every nonnegative value precisely once. Hence it is a univalent function in the circular ring which maps this domain onto the complex plane slit along two segments of the negative axis. In particular, the segment corresponding to the circumference $|z|=r$ begins at the origin.

The geometric properties just described identify $\left[z k^{\prime}(z)\right]^{2}$ up to a factor as

$$
\left[z k^{\prime}(z)\right]^{2}=\frac{A}{\wp(\theta)-\wp(\phi)},
$$

where $\theta=\log z / r$ and $\phi=\log b / r$ and $\wp(\theta)$ is the Weierstrass $\wp$-function of period $\log 1 / r$ and $i \pi$ (see, for example, [7, p. 191]).

7. Solving for $J$ yields

$$
J[f(z)]=\frac{K+1}{2}[k+\bar{k} Q]
$$

where $J$ is the elliptic integral defined in (16) and $Q=\sqrt{(K-1) /(K+1)}$.

After inverting (19) we will have an explicit expression for the extremal function

$$
f(z)=P\left\{\frac{K+1}{2}(k(z)+\overline{k(z)} Q)\right\},
$$

where $P$ is closely related to the Weierstrass $\wp$-function. 
8. Although the analytic function $k(z)$ is determined only up to a factor, various relationships can be used in order to determine the extremal value $B$. The extremal function is explicitly expressed in (20) as the inverse of the elliptic integral (16). The periods of (20) are functions of $B$. The branch points of (16) are real.

From (1) it follows that as $z$ traverses the unit circle, $w$ does the same. By Cauchy's integral theorem

$$
\int_{|w|=1} \frac{d w}{\sqrt{w(B-w)(1-B w)}}=2 \int_{0}^{B} \frac{d w}{\sqrt{w(B-w)(1-B w)}}=2 \omega_{1} \text {. }
$$

The right-hand side of $(21)$ is twice the real period of the elliptic integral (16). Consider equation (15) for $|z|=1$. Since $d k$ is real on that circumference, we find that

$$
\int_{|z|=1} k^{\prime}(z) d z=2(1-Q) \omega_{1}
$$

The imaginary period is

$$
\omega_{2}=\int_{B}^{1 / B} \frac{d w}{\sqrt{w(B-w)(1-B w)}} .
$$

Since

$$
\int_{B}^{1} \frac{d w}{\sqrt{w(B-w)(1-B w)}}=\int_{1}^{1 / B} \frac{d w}{\sqrt{w(B-w)(1-B w)}},
$$

we have $\frac{1}{2} \omega_{2}=\int_{B}^{1} d w / \sqrt{w(B-w)(1-B w)}$. From (15) we have

$$
\int_{b}^{1} d k=\int_{B}^{1} d J-Q \int_{B}^{1} \overline{d J}=\frac{1}{2} \omega_{2}+\frac{Q}{2} \omega_{2}
$$

from which follows

$$
\int_{b}^{1} k^{\prime}(z) d z=\frac{1}{2}(1+Q) \omega_{2}
$$

The left sides of (22) and (25) are known up to a factor. The quotient of (25) by (22) is thus a completely determined function of $b$. Denote the quotient by $E(b)$. The ratio of the periods is then

$$
4 E(b) \frac{(1-Q)}{(1+Q)}=\frac{\omega_{2}}{\omega_{1}}=\tau .
$$

9. Since the ratio $\tau$ of the periods is known from (26), it is possible to express the extremal value $B$ as a function of the fixed point $b$. This can be done as follows.

Putting the elliptic integral (16) in the Weierstrass normal form, we have 


$$
\int \frac{d w}{\sqrt{w(B-w)(1-w B)}}=\int \frac{d w}{\sqrt{4\left(w-e_{1}\right)\left(w-e_{2}\right)\left(w-e_{3}\right)}}
$$

where $e_{1}+e_{2}+e_{3}=0$.

The result of a straightforward calculation is

$$
B^{2}=\frac{e_{2}-e_{3}}{e_{1}-e_{3}} \text {. }
$$

But the right side is the elliptic modular function $\lambda(\tau)$ which is a single valued function of $\tau,[15],[16]$, which in turn is a function of $b$.

\section{REFERENCES}

1. L. V. Ahlfors, Lectures on quasiconformal mappings, Van Nostrand, Princeton, N.J., 1966.

2. P. L. Duren and M. M. Schiffer, $A$ variational method for functions schlicht in an anmulus, Arch. Rational Mech. Anal. 9 (1962), 260-272.

3. D. Gaier, Untersuchungen zur Durchführung der konformen Abbildung mehrfach zusammenhängender Gebiete, Arch. Rational Mech. Anal. 3 (1959), 149-178.

4. D. Gaier and F. Huckemann, Extremal problems for functions schlicht in an annulus, Arch. Rational Mech. Anal. 9 (1962), 415-421.

5. F. W. Gehring and G. Hälström, $A$ distortion theorem for functions univalent in an annulus, Ann. Acad. Sci. Fenn. Ser. A I 325 (1963).

6. H. Grötzsch, Uber einige extremal Probleme der konformen Abbildung, Leipziger Berichte 80 (1928), 367-376.

7. H. Kober, Dictionary of conformal representations, Dover, New York, 1957.

8. O. Lehto and K. Virtanen, Quasiconformal mappings in the plane, Springer-Verlag, New York, 1973.

9. F. Marty, Sur le module des coefficients de Maclaurin d'une fonction univalente, C. R. Acad. Sci. Paris 198 (1934), 1569-1571.

10. H. Renelt, Modifizierung und Erweiterung einer Schifferschen Variationsmethode für quasikonforme Abbildungen, Math. Nachr. 55 (1973), 353-379.

11. M. Schiffer, $A$ method of variations within the family of simple functions, Proc. London Math. Soc. 44 (1938), $432-449$.

12. _ A variational method for univalent quasiconformal mappings, Duke Math. J. 33 (1966), 395-411.

13. M. Schiffer and G. Schober, An extremal problem for the Fredholm eigenvalues, Arch. Rational Mech. Anal. 44 (1971/72), 83-92.

14. _ A remark on the paper "An extremal problem for the Fredholm eigenvalues," Arch. Rational Mech. Anal. 46 (1972), 394.

15. J. Tannery and N. Molk, Elements de la théorie des fonctions elliptiques, Gauthier-Villars, Paris, 1902.

16. E. T. Whittaker and G. N. Watson, $A$ course of modern analysis, Cambridge Univ. Press, New York, 1946.

Department of Mathematics, Harvey Mudd College, Claremont, California 91711 